\title{
Numerical modeling in photonic crystals integrated technology: the COPERNICUS Project
}

\author{
Stefania Malaguti, \\ Andrea Armaroli, \\ Gaetano Bellanca, \\ Stefano Trillo \\ Department of Engineering \\ University of Ferrara, Italy
}

Yannick Dumeige, Mathilde Gay

UEB, CNRS-FOTON, University of Rennes 1, Lannion, France

\author{
Simeon Kaunga-Nyirenda, \\ Jun Lim, Eric Larkins \\ Department of Electrical \\ and Electronic Engineering, \\ University of Nottingham, \\ Nottingham, UK
}

\author{
Philip Trøst Kristensen, \\ Kresten Yvind, \\ Jesper Mørk \\ Department of Photonics Engineering, \\ Technical University of Denmark, \\ Lyngby, Denmark
}

\begin{abstract}
Photonic crystals will play a fundamental role in the future of optical communications. The relevance of the numerical modeling for the success of this technology is assessed by using some examples concerning the experience of the COPERNICUS Project.
\end{abstract}

\section{INTRODUCTION}

Photonic crystals are periodic dielectric nanostructures that permit to control the light flow at sub-micrometer scales. They exhibit disrupting capabilities of routing, switching, and multiplexing in an ultra-compact integrated format and will play a key role in the future of optical communications and interconnects. COPERNICUS (Compact Otdm/wdm oPtical rEciveRs based on photoNic crystal Integrated CircUitS) is a European project whose aim is to develop a cutting-edge photonic crystal technology for the high density integration of basic optical functions. The scientific and technological objectives of COPERNICUS target the development of compact demultiplexing receivers for $100 \mathrm{~Gb} / \mathrm{s}$ optical time division multiplexed (OTDM) and wavelength division multiplexed (WDM) signals, including high-speed integrated photodetectors. Coordinated by Thales Research and Technology (TRT), France, the Consortium brings together different academic and industrial partners with high profiles in photonics, nanotechnology, modeling and developing new technologies for telecommunications and aerospace (the University of Nottingham UK (UNott); the Laboratory for Photonics and Nanostructures (LPN) and the Laboratory of Optical Functions for Information and Communication Technologies (FOTON), both research units of the French National Center of Scientific Research (CNRS); DTU Fotonik at the Technical University of Denmark; the University of Ferrara (UniFe) in Italy and industrial partners u2t Photonics (U2T), Germany and Thales Systèmes Aéroportés (TSA), France). The aim of this paper is to highlight the pivotal role of numerical modeling in the development of this disruptive technology.
Pierre Colman, Sylvain Combrié, Alfredo De Rossi

Thales Research and Technology, Palaiseau, France

\section{THE COPERNICUS'S EXPERIENCE}

The Project focuses on the development and integration of photonic crystal devices for OTDM and WDM receivers. The design of the different components is addressed by means of several techniques such as, for example, Coupled Mode Theory (CMT), the Finite Difference in the Time Domain (FDTD), etc. For brevity, only some examples are reported here. More details can be found on the project website (http://www.copernicusproject.eu).

\section{A. OTDM Receiver}

The realization of an all-optical time domain receiver operating at $100 \mathrm{~Gb} / \mathrm{s}$ is a real challenge. In this component, the extraction of the output signal from the input frame is obtained by means of an optical control pulse. Nonlinear effects in a nanocavity system permit to achieve this operation [1]. Material and structural properties influence each other and require a thorough understanding of a complex dynamical evolution as well as an estimation of physical properties (e.g. carrier lifetime). Design procedures are performed by different partners (DTU, TRT, UniFe) whereas LPN leads the activity on material engineering, which is required to manipulate the carrier lifetime of the active medium. Fabrication is performed at DTU and TRT and final characterization at both FOTON and TRT. As an example, fig. 1 shows a schematic representation of the three-ports AOG and the simulated evolution of a signal pulse at different pump levels.

\section{B. WDM Receiver}

The second key component for COPERNICUS is a four channel WDM receiver operating at $25 \mathrm{~Gb} / \mathrm{s}$ per channel. The core of this device is a wavelength drop filter. As an example, the layout of a two stage filter (operating at $\lambda_{1}=1.46 \mu \mathrm{m}$ and $\lambda_{2}=1.48 \mu \mathrm{m}$ respectively) is shown on fig. 2 (right). Each stage is realized with two L3 coupled cavities [2]. A reliable filter is obtained by the careful adjustment of structural 


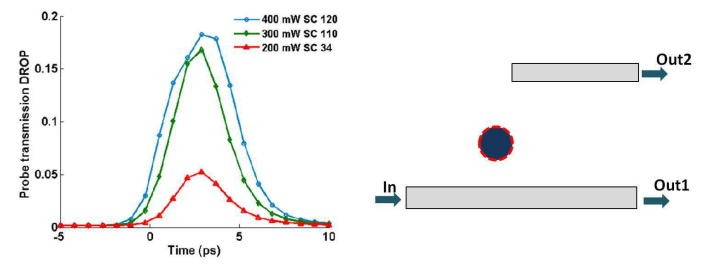

Fig. 1. Schematic representation of the AOG (right). Pump-probe operations in a three-ports AOG optimized design (left). The curves show the probe efficiency at the drop port for different control power injected into the structure. The corresponding values of the switching contrast (SC) are also indicated.

parameters, such as the hole positions and radii. The left part of this figure reports the reflection $\left(S_{11}\right)$ and transmission coefficients for the through port $\left(S_{21}\right)$ and the two drop ports $\left(S_{31}\right.$ and $\left.S_{41}\right)$. The final device with four channels is realized by duplicating this configuration. The design has been performed by UniFe, fabrication by DTU, LPN and TRT. Final measurements will be made at both TRT and FOTON.

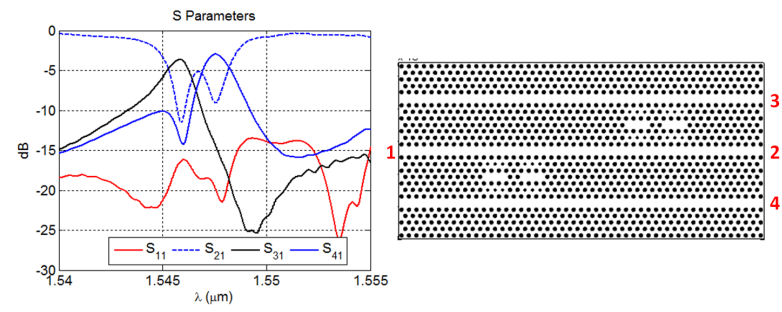

Fig. 2. Sketch of a two stage filter realized with two coupled L3 cavities (right) and $\mathrm{S}$ parameters of the filter (left).

\section{Photodetector}

Electronic output is provided by a compact integrated photodetector. The requirements for the photodetector are high responsivity $(>0.5 \mathrm{~A} / \mathrm{W})$ and fast response $(>28 \mathrm{GHz})$. This presents a number of design challenges. For example, a vertical PIN structure with an absorbing layer must be integrated with the $\mathrm{PhC}$ waveguide. The bandwidth requires devices with a small RC time constant, so the junction area must be kept small. For high responsivity, the optical field must be transmitted from a single-mode $\mathrm{PhC}$ waveguide into the small photodetector, requiring careful optical matching and efficient lateral optical confinement. Optical and electrical simulations have been performed using FDTD (UniFe) and bipolar electro-thermal models (UNott), respectively. The electric field dependence of the carrier velocity is taken into account using a field-dependent mobility model. Fig. 3 shows that the constant low-field mobility overestimates the modulation response at high reverse bias. The use of field dependent carrier mobilities gives good agreement with published experimental results [3].

\section{Photonic Crystal Circuitry}

The different key components of each device must be properly connected by means of single mode optical "circuitry" integrated on the same chip. Examples of circuit components

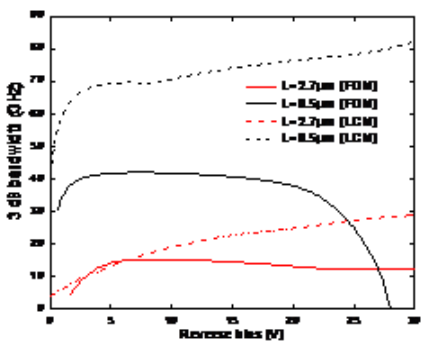

Fig. 3. Frequency response of the photodetector. LCM: constant low-field mobility model (dashed lines), FDM: field-dependent mobility model (solid lines).

are signal splitters, bends and delay lines. To optimize the layout, DTU and UniFe have set-up a design procedure based on 3D-FDTD and Topological Optimization (TO) [4]. This procedure has been successfully applied to the design of a Y-junction and a double $60^{\circ}$ bend, whose optimized layout is illustrated in fig. 4 (right). The same figure (left) show the transmission $\left(S_{21}\right)$ and the reflection $\left(S_{11}\right)$ coefficients for the optimized (continuous lines) and the unoptimized (dashed lines) configurations; the impressive positive effects of TO are evident.
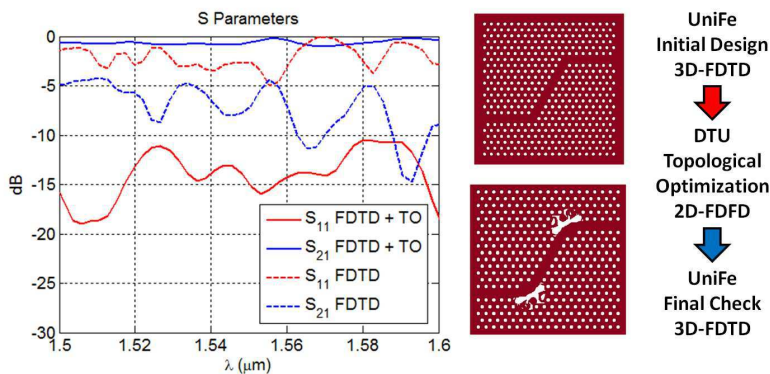

Fig. 4. Optimization procedure for the design of a double $60^{\circ}$ bend (right) with the final layout of the device. Transmission $\left(S_{21}\right)$ and reflection $\left(S_{11}\right)$ coefficients (left) for the optimized (continuous lines) and the unoptimized (dashed lines) configurations.

\section{CONCLUSIONS}

The ground-breaking capabilities of $\mathrm{PhC}$ circuits and systems demand a comprehensive design strategy in order to promptly provide reliable design guidelines. The ambitious goals of the COPERNICUS project are pursued by means of different tools according to a multiphysics approach.

\section{ACKNOWLEDGMENT}

The authors acknowledge the contribution of all the other partners for this work, as well as funding from the EC under the project Copernicus (249012).

\section{REFERENCES}

[1] S. Combrié, C. Husko, P. Colman, Q.V. Tran, F. Raineri, A. De Rossi, ECOC 2009, 20-24 Sept., 2009.

[2] B.K. Min, J.E. Kim, H.Y. Park, Opt. Comm., 237,59-63, (2004)

[3] J.E. Bowers, C. A. Burrus, J. Lightwave Technol., 5, 13391350, (1987)

[4] L.H. Frandsen, A. Harpoth, P.I. Borel, M. Kristensen, J.S. Jensen, and O. Sigmund, Opt. Express 12, 5916-5921, (2004). 